\title{
Proposal of a prognostically relevant grading scheme for pulmonary squamous cell carcinoma
}

\author{
Wilko Weichert ${ }^{1,2,3}$, Claudia Kossakowski ${ }^{1}$, Alexander Harms ${ }^{1}$, \\ Peter Schirmacher ${ }^{1}$, Thomas Muley ${ }^{4,5}$, Hendrik Dienemann ${ }^{5,6}$ and Arne Warth ${ }^{1,5}$
}

Affiliations: ${ }^{1}$ Institute of Pathology, Heidelberg University, Heidelberg, Germany. ${ }^{2}$ National Center for Tumor Diseases (NCT), Heidelberg, Germany. ${ }^{3}$ Institute of Pathology, Technical University Munich (TUM), Munich, Germany. ${ }^{4}$ Translational Research Unit, Thoraxklinik at Heidelberg University, Heidelberg, Germany. ${ }^{5}$ Translational Lung Research Center Heidelberg, Member of the German Center for Lung Research, Heidelberg, Germany. ${ }^{6}$ Dept of Thoracic Surgery, Thoraxklinik at Heidelberg University, Heidelberg, Germany.

Correspondence: Wilko Weichert, Institute of Pathology, Heidelberg University, INF 224, 69120 Heidelberg, Germany. E-mail: wilko.weichertamed.uni-heidelberg.de

ABSTRACT Recent studies in lung adenocarcinoma established a clinically relevant histomorphologybased classification. In contrast, no morphological classifiers have yet been implemented into routine diagnostics for lung squamous cell carcinoma (SQCC). However, morphology-based characteristics putatively impacting on survival have been proposed.

We analysed a cohort of 541 SQCC patients with complete clinical follow-up data for morphological characteristics (keratinisation, tumour cell budding, size of tumour cell nests, nuclear size and stromal content). Morphological characteristics were correlated with clinical data and patient outcome.

Keratinisation, budding, stromal content and tumour cell nest size, but not nuclear size, were associated with distinct clinicopathological characteristics and survival. SQCC patients with keratinisation, small cell nest size, high stromal content and extensive budding had shorter overall survival. A combined grading scheme composed of the two most reliable validated prognostic markers, i.e. budding and nest size, resulted in an age-, stage- and sex-independent prognosticator for overall survival with a hazard ratio of 1.6 for grade 2 tumours and a hazard ratio of 3.7 for grade 3 tumours when compared with grade 1 neoplasms $(\mathrm{p}<0.001)$.

Morphological characteristics of SQCC have significant prognostic impact and could constitute the basis for a diagnostically relevant future SQCC grading scheme.

@ERSpublications

Morphological characteristics of pulmonary squamous cell carcinoma have significant prognostic impact for survival http://ow.ly/St6mM

Editorial comment in: Eur Respir J 2016; 47: 720-723 [DOI 10.1183/13993003.00035-2016].

This article has supplementary material available from erj.ersjournals.com

Received: June 162015 | Accepted after revision: Sept 182015 | First published online: Nov 052015

Conflict of interest: Disclosures can be found alongside the online version of this article at erj.ersjournals.com

Copyright OERS 2016 


\section{Introduction}

Numerous international studies have validated the prognostic value of the morphology-based International Association for the Study of Lung Cancer/American Thoracic Society/European Respiratory Society classification of pulmonary adenocarcinoma (ADC) [1] and even further morphological refinements might be of relevance in ADC with respect to an optimised prognostic patient stratification [2-5]. Thus, semi-quantitative assessment of histomorphology is now the standard for ADC and the backbone of the 2015 World Health Organization (WHO) classification of these tumours [6].

In contrast, only little progress has been made with respect to the use of histomorphology as a prognosticator in lung squamous cell carcinoma (SQCC) and subtyping according to the 2004 WHO classification [7] has not been demonstrated to be of any clinical relevance.

However, KADOTA et al. [8] recently began to address this problem and analysed a series of 485 resected SQCCs for specific morphological and cytological criteria. They demonstrated that overall survival was significantly decreased in SQCCs with single-cell invasion, large nuclei and with high tumour budding activity. In order to challenge and expand this dataset, and to pave the way towards a clinically relevant grading system, we retrospectively analysed a large cohort of surgically resected SQCCs with complete follow-up for a morphological parameter set including those putative prognostic stratifiers suggested by KADOTA et al. [8].

\section{Materials and methods \\ Cohort}

Our cohort consisted of 541 SQCCs which were completely resected between 2002 and 2010 at the Thoraxklinik Heidelberg. Six patients (1.1\%) received wedge resection, seven patients $(1.3 \%)$ were treated with segmentectomy, 360 patients had lobectomy (66.5\%), 23 patients received bilobectomy (4.3\%) and 145 patients were treated with pneumonectomy (26.8\%), in most cases accompanied by systematic lymph node dissection. Histologies other than pure SQCC were excluded. None of the patients received neoadjuvant radio- and/or chemotherapy. 442 (81.7\%) patients were male and 99 (18.3\%) were female. Mean age at diagnosis was 64.8 years (range 38.2-82 years). 112 (20.7\%) patients received adjuvant chemotherapy and $68(12.6 \%)$ patients were treated with adjuvant mediastinal radiation following the national guidelines in effect at the time of diagnosis. 180 (33.3\%) patients died during follow-up and 162 (29.9\%) relapsed. Mean follow-up time of patients alive at the end-point of analysis was 40.8 months. Mean follow-up time of patients without recurrence at the end-point of analysis was 37.1 months. The distribution of clinicopathological variables is given in table 1 .

\section{Histologic evaluation}

All archived haematoxylin and eosin-stained slides were reviewed by two experienced pathologists (C.K. and A.W.) following the criteria described by KADOTA et al. [8] using an Olympus BX51 microscope. Tumours were classified as nonkeratinising, keratinising or basaloid SQCC in accordance with the 2015 WHO classification (online supplementary figure S1) [6]. Tumour budding and the size of the smallest tumour nest were assessed at the areas showing the most extensive budding activity and the maximal number of small tumour nests [8].

Tumour budding was defined as small tumour nests composed of $<5$ tumour cells (separately analysed in one high-power field (HPF; $\times 400$ ) with the highest number of budding and as total budding in 10 HPFs) which "branch" away from larger nests and "bud" into the adjacent parenchyma (online supplementary figure S1). With the applied microscope setting, one HPF corresponds to a field diameter of $0.55 \mathrm{~mm}$ $\left(0.24 \mathrm{~mm}^{2}\right)$.

A cell nest was defined as a cluster of tumour cells surrounded by tumour stroma. The size of the smallest invasive tumour nest was further classified into large nests (composed of $>15$ tumour cells), intermediate nests (5-15 tumour cells), small nests (2-4 tumour cells) and single-cell invasion (online supplementary figure S1). The size of the smallest tumour nest was assessed in two categories: 1) the smallest tumour nest in the entire sampled tumour area and 2) the smallest tumour nest at the invasion front.

Tumour stromal content was categorised as low ( $\leqslant 25 \%$ of the whole tumour area), moderate $(26-50 \%)$ or high $(>50 \%)$ (online supplementary figure S1). For assessment of the nuclear diameter, tumour areas with the largest nuclei were selected. We analysed the average nuclear diameter of at least 100 tumour cells using nearby small lymphocytes as reference. Small nuclei were defined as having a diameter of three lymphocytes or less, intermediate nuclei corresponded to the diameter of approximately four lymphocytes and large nuclei were defined as measuring more than four lymphocytes. Proliferative activity was assessed by Ki-67 immunohistochemistry as previously described [9]. 
TABLE 1 Survival associations for clinicopathological factors including morphological characteristics of the SQCCS

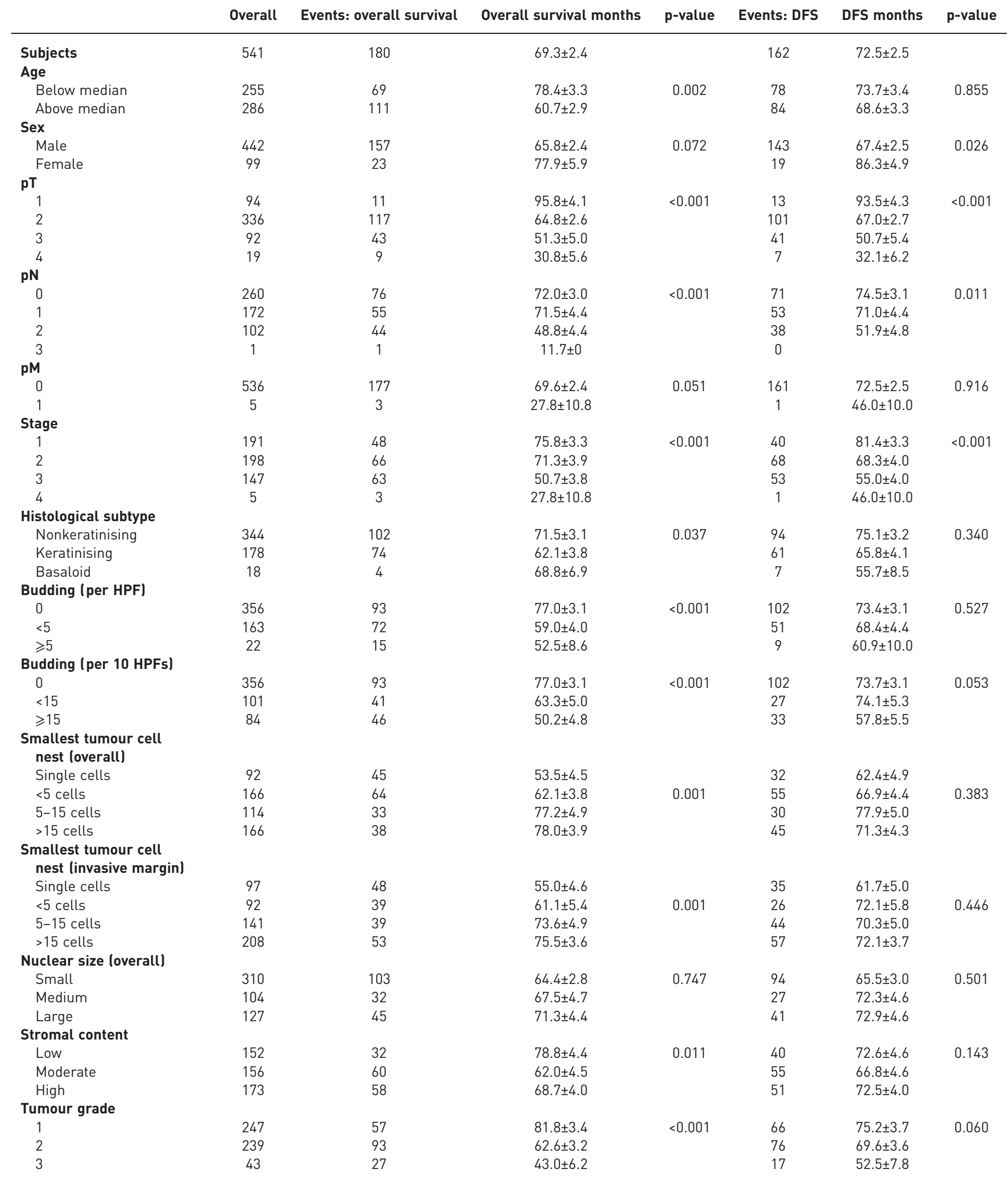

Data are presented as $\mathrm{n}$ or mean \pm SE, unless otherwise stated. SQCC: squamous cell carcinoma; DFS: disease-free survival; $\mathrm{pT}$ : primary tumour; pN: regional lymph nodes; pM: distant metastasis; HPF: high-power field. Not all parameters could be assessed in all patients. 


\section{Results}

Morphological characteristics of SQCCS

One-third of the SQCCs $(178 / 541,33 \%)$ showed keratinisation and 63.7\% (344/541) were nonkeratinising SQCCs. Basaloid SQCCs (18 cases; 3.3\%) were rare (table 1).

Tumour cell budding at the invasive margin was observed in 185 (34.2\%) out of 541 cases. Of these, only 22 cases $(4.1 \%)$ had $>5$ budding foci per single HPF. A comparable budding rate (185 out of 541 cases, $34 \%)$ was demonstrated when a larger part (10 HPFs) of the invasion front was investigated. However, when the number of total budding foci in $10 \mathrm{HPFs}$ was evaluated and a slightly different cut-off of 15 foci was applied, 84 cases (15.5\%) fell in the category of tumours with enhanced budding activity (table 1).

In $92(17.1 \%)$ cases we observed single-cell invasion. 166 (30.9\%) cases had tumour cell nests composed of $<5$ tumour cells, 114 tumours (21\%) fell in the category with a minimum cell nest size of 5-15 cells, while 166 tumours $(31 \%)$ were exclusively composed of large tumour cell nests containing $>15$ cells. When only the invasive margin was considered, the rate of SQCC with single-cell invasion or small nests $(<5$ cells $)$ was slightly lower (combined $34.9 \%$ compared with combined $48 \%$; table 1).

310 (57.3\%) out of 541 SQCCs featured small nuclei; the rest of the cases were distributed quite equally between intermediate $(104,19.2 \%)$ and large $(127,23.5 \%)$ nuclear sizes (table 1). The amount of stromal content was low, moderate and high in 152 (31.6\%), $156(32.4 \%)$ and $173(36 \%)$ of the cases, respectively.

\section{Correlation of morphological characteristics in SQCCS}

The presence of keratinisation was significantly linked to tumour cell budding and the size of the tumour cell nests. SQCCs with keratinisation and basaloid morphology tended to show slightly higher budding frequencies when compared with nonkeratinising SQCCs (online supplementary figure S2). Tumours with smaller cell nests (overall and at the invasive margin) were more frequent in the keratinising subgroup when compared with the nonkeratinising and basaloid subgroups (online supplementary figure S2). Nuclear size was not associated with the histological subtype of SQCCs. As expected, maximum tumour cell budding in one HPF and total budding in $10 \mathrm{HPFs}$ as well as overall tumour cell nest size and tumour cell nest size at the invasive margin were significantly associated. SQCCs with small cell nest sizes had a higher propensity towards stronger budding activity. Furthermore, SQCCs with higher budding activity and smaller nest sizes were significantly more likely to feature large nuclei and a higher stromal content (online supplementary table S1).

\section{Correlation of morphological characteristics of SQCCS with clinical parameters}

Patients with keratinising SQCCs were younger ( $\mathrm{p}=0.004$; online supplementary table S2) when compared with patients with nonkeratinising SQCCs. Other significant associations of keratinisation with clinicopathological characteristics were not observed.

The presence of tumour cell budding was positively associated with the primary tumour ( $\mathrm{pT}$ ) category $(\mathrm{p}=0.002)$, while further clinicopathological associations were not evident (figure 1 and online supplementary table S2).

Cell nest size (overall and at the invasive margin) was strongly linked to clinicopathological variables. Locally advanced tumours with nodal positivity exhibited smaller cell nests when compared with their locally restricted, node-negative counterparts (figure 1 and online supplementary table S2). An association of nodal positivity with high stromal content was also noted (online supplementary table S2). Nuclear size did not show associations with clinicopathological parameters.

\section{Correlation of morphological characteristics with proliferative activity}

Basaloid morphology was strongly associated with high proliferative activity (Ki-67: 63\%). Proliferative activity did not differ between nonkeratinising (47\%) and keratinising SQCCs (48\%; p=0.021). We did not observe significant correlations between proliferative activity and budding, cell nest size or nuclear diameters. The Ki-67 proliferation index was significantly higher in tumours with low stromal content (online supplementary table S2).

\section{Correlation of morphological characteristics of SQCCS with survival}

Patients with keratinising SQCCs had a shortened overall survival (62.1 months) when compared with patients with nonkeratinising (71.5 month) and basaloid SQCCs (68.8 months; $\mathrm{p}=0.037$; table 1 and online supplementary figure S3). This linkage with survival was retained in multivariate analysis under inclusion of age, sex and stage (online supplementary table S3). Compared with nonkeratinising SQCCs, keratinising SQCCs had a hazard ratio of $1.55(\mathrm{p}=0.011)$. Associations of the histologic type with disease-free survival (DFS) were not evident. To elucidate the unexpected good survival of basaloid SQCCs, we addressed if this 

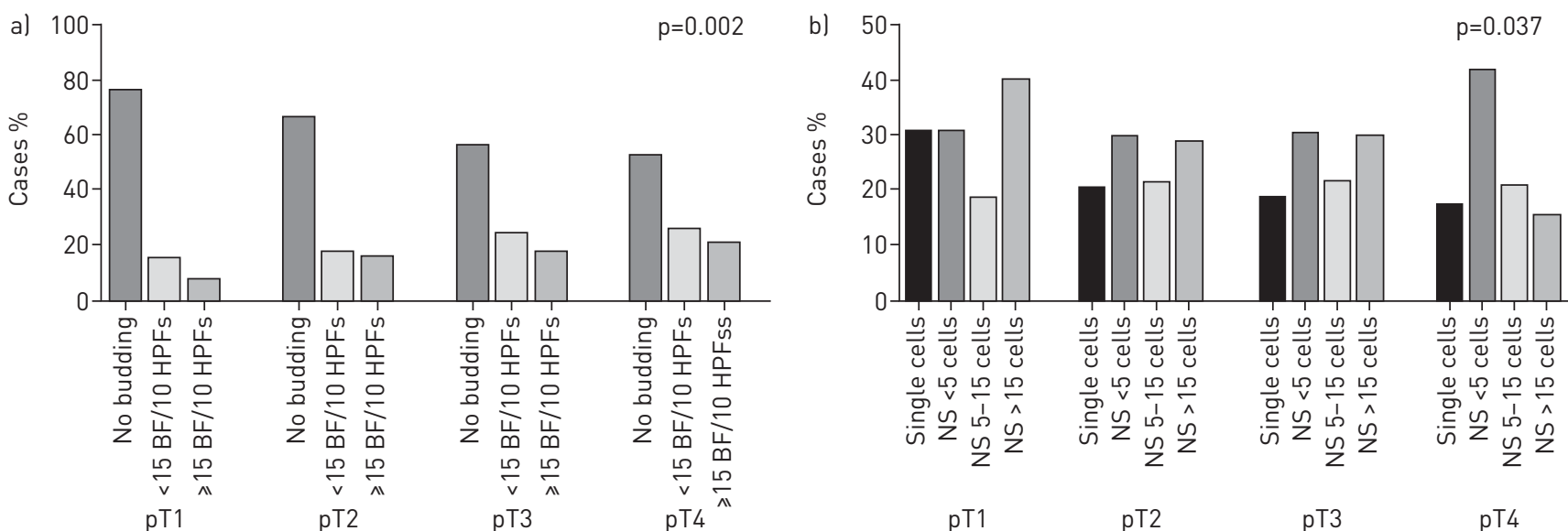
pT1
pT2
pT3
pT4
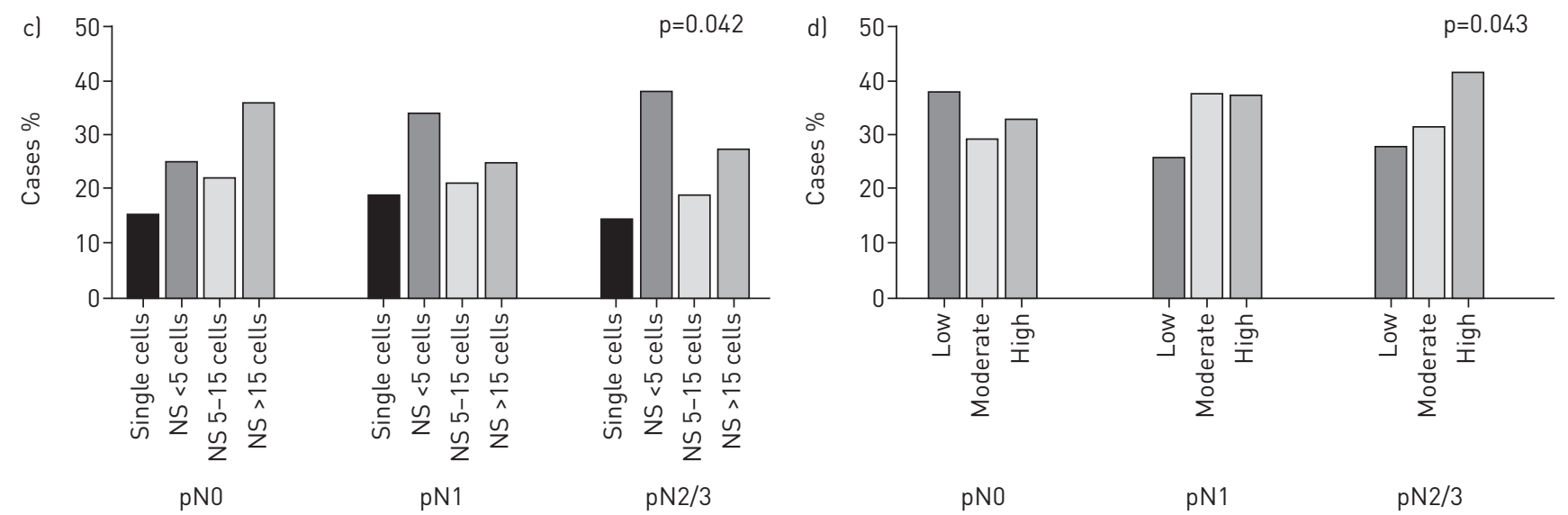

FIGURE 1 Exemplary associations of clinical parameters with morphology. Association between local tumour extent (pT: primary tumour) and a) tumour cell budding at the invasive margin as well as b) overall tumour nest size. Association between nodal spread (pN: regional lymph nodes) and c) overall tumour nest size and d) stromal content. BF: budding foci; HPF: high-power field; NS: nest size.

was potentially due to a beneficial effect of adjuvant chemotherapy in this group. While all three patients with basaloid SQCCs who received adjuvant chemotherapy survived despite higher pT stages (pT2a, pT2b, pT4), patients with basaloid SQCCs who did not receive adjuvant chemotherapy had a survival comparable to patients with nonkeratinising SQCCs (online supplementary figure S4).

Tumour cell budding had high impact on overall survival both when evaluated in one HPF and in 10 HPFs. Patients with $\geqslant 15$ budding foci per $10 \mathrm{HPFs}$ had a mean overall survival of 50.2 months, compared with 63.3 months for those patients whose tumours had $<15$ budding foci and 77.0 months for those patients whose tumours displayed no budding foci at all $(\mathrm{p}<0.001$; figure 2 and table 1). Survival associations were comparable when only one HPF was evaluated (online supplementary figure S5 and table 1). Again, this effect was independent of age, sex and stage. Compared with patients without tumour cell budding in $10 \mathrm{HPFs}$, patients with $<15$ foci had a hazard ratio of 1.6 and patients with $\geqslant 15$ foci had a hazard ratio of $2.4(\mathrm{p}<0.001$; online supplementary table S4). Budding per $10 \mathrm{HPFs}$ had a borderline association with DFS ( $\mathrm{p}=0.053$; online supplementary figure $\mathrm{S} 5$ and table 1$)$. When performing a probatory multivariate analysis, the slight impact on DFS was preserved independent of age, sex and stage, but hazard ratios were only elevated in the group of tumours with $\geqslant 15$ budding foci (hazard ratio 1.6; $\mathrm{p}=0.033$ ), but not in the group with $<15$ foci.

The size of tumour cell nests was strongly associated with overall survival. The longest survival times (mean 78.0 months) were noted in patients with overall large cell nest sizes ( $>15$ cells), while with decreasing cell nest sizes overall survival decreased as well (5-15 cells: 77.2 month; $<5$ cells: 62.1 months; single cells: 53.5 months; $\mathrm{p}=0.001$; figure 2 and table 1). For tumour cell nest size at the invasion front, comparable survival associations were seen (online supplementary figure S6 and table 1). The survival 

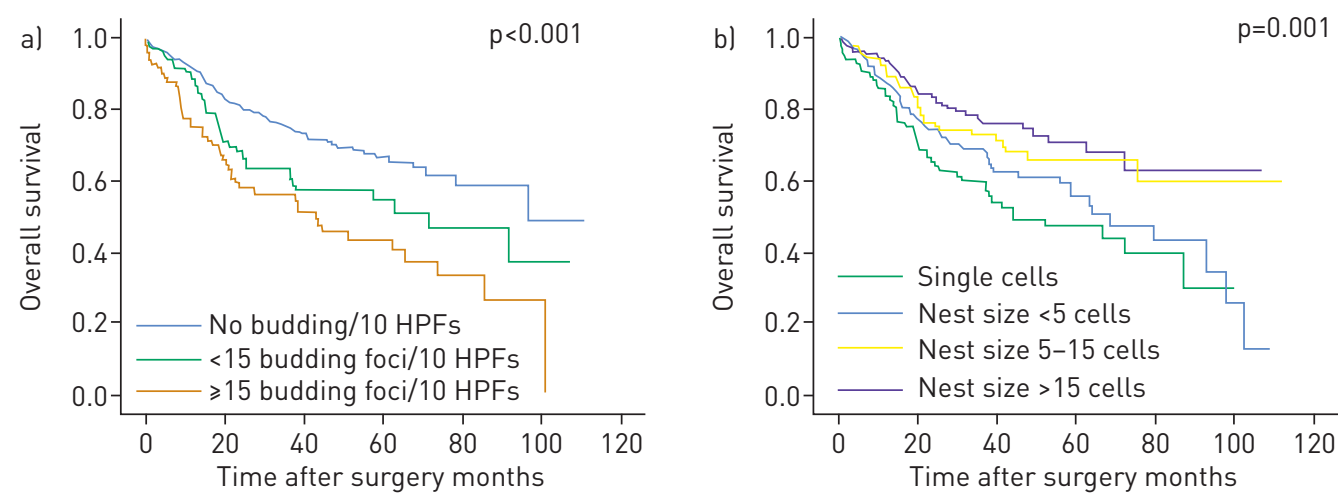

FIGURE 2 Association of budding activity and nest size with survival in squamous cell carcinoma. Patients were stratified for a) tumour cell budding at the margin (per 10 HPFs) as well as for b) overall tumour cell nest size. HPF: high-power field.

impact of both parameters was independent of age, sex and stage. Compared with patients with single-cell invasion (hazard ratio 1), patients with an overall tumour cell nest size of $<5$ cells had a hazard ratio of 0.72 , which was further decreased to 0.56 and 0.44 for patients whose tumour nests contained 5-15 or $>15$ cells, respectively ( $\mathrm{p}=0.002$; online supplementary table S5). A significant association of tumour cell nest size with DFS was not evident (online supplementary figure S6 and table 1).

Nuclear size had no impact on prognosis in our cohort (table 1 and online supplementary figure S7). With respect to the stromal content, tumours with moderate and high stromal content had a significantly worse overall survival compared with tumours with low stromal content $(p=0.011)$; this effect was not evident for DFS (table 1 and online supplementary figure S7).

\section{Grading of lung SQCC}

According to currently available data there seems to be no one dominating histological classifier which can solely be used to determine tumour grade in SQCC. Therefore, a combination of parameters appears to be necessary to assign a clinically relevant grade. To this end, to keep it simple, we focussed on the factors with the highest survival impact, which have been proposed by KADOTA et al. [8] and could be independently validated by us (tumour budding per $10 \mathrm{HPFs}$ and overall nest size). We decided to leave the other parameters out as they were either new (stromal content), redundant (tumour budding per one HPF, nest size at the margin) or results were conflicting with previous work (nuclear size, histological subtype). After assigning a score for budding (1: no budding/10 HPFs; 2 : $<15$ budding foci/10 HPFs; $3: \geqslant 15$ budding foci/ $10 \mathrm{HPFs}$ ) and for nest size (1: $>15$ cells; $2: 5-15$ cells; $3:<5$ cells; 4 : single cells) we calculated an overall grading score (range $2-7$ ) for every case by summing up the two variables. The respective survival curves for the raw scores resulted in an exceptionally good delineation of discrete prognostic groups for overall survival ( $p<0.001$; online supplementary figure S8). For DFS only the maximal score of 7 defined a group with an exceptionally poor prognosis (online supplementary figure S8). On the basis of these data we defined tumours with a score of 2 or 3 as being well differentiated (grade 1), tumours with a score of 4-6 as moderately differentiated (grade 2) and tumours with the maximal score of 7 as being poorly differentiated (grade 3). This resulted in 247 (47\%) grade 1 cases, 239 (45\%) grade 2 cases and 43 (8\%) cases assigned to grade 3 (table 1). High-grade tumours tended to be more locally advanced; other clear-cut associations with clinical parameters were not noted (online supplementary table S2). A delineation of overall survival according to our grading scheme resulted in a clear-cut separation of prognostic groups (figure 3 and table 1). Grade 3 was also associated with significantly poorer DFS; however, grade 1 and grade 2 tumours behaved similar in this regard (online supplementary figure S9 and table 1). Multivariate survival analysis under inclusion of grade, age, sex and stage confirmed the strong independent prognostic impact of grade on overall survival in SQCC. Grade 2 and 3 tumours had a hazard ratio of 1.6 and 3.7, respectively, when compared with grade 1 neoplasms $(\mathrm{p}<0.001$, table 2$)$.

\section{Discussion}

We analysed the prognostic impact of different histomorphological characteristics in a large cohort of 541 resected SQCCs. We demonstrate that keratinisation, budding, single-cell invasion and higher stromal content are associated with a significantly worse overall survival. Out of the two most reliable predictors, we derived a simple grading scheme with strong independent prognostic impact. 


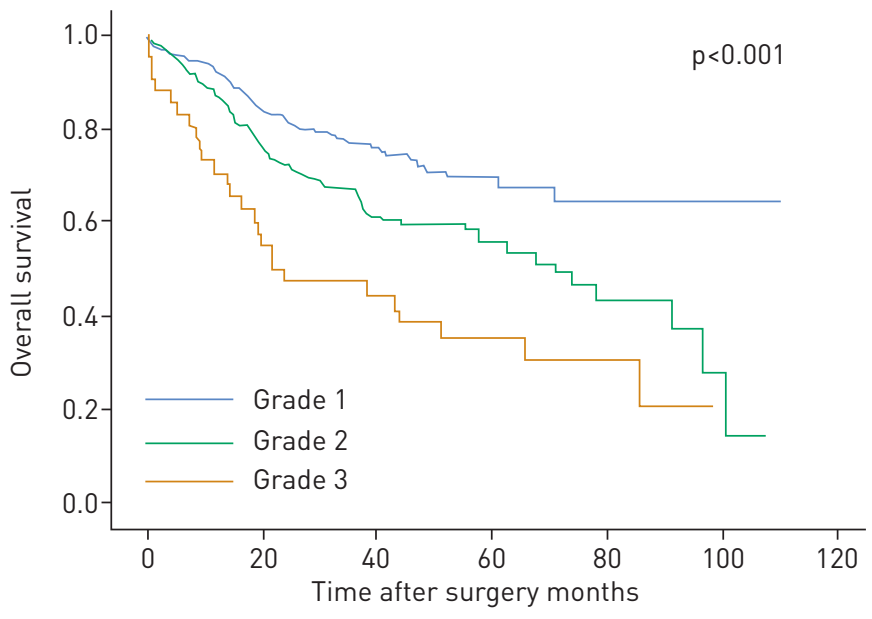

FIGURE 3 Association of the new squamous cell carcinoma tumour grade with overall survival.

Up to now there have been no established histomorphological characteristics of SQCC that are used in routine diagnostics for prognostic patient stratification. The 2015 WHO classification separates SQCC into keratinising, nonkeratinising and basaloid tumours. Grading is not recommended [6].

From other tumour entities, such as colorectal cancer [10], it is known that single-cell invasion and tumour budding might be associated with a poor prognosis, a phenomenon which has recently also been described for pulmonary SQCC [8] and ADC [11]. In addition, another exploratory study provided evidence for a prognostic impact of single-cell invasion in pulmonary SQCC [12]. Likewise, tumour nest size has also been suggested as a prognostic factor in related tumour entities such as head and neck SQCC [13].

So far, SQCCs have usually been graded according to a decreasing degree of keratinisation into well, moderately and poorly differentiated tumours. However, data on the impact of keratinisation on survival in SQCC are limited in the published literature and a definite proof that keratinisation has a survival impact in this tumour entity is missing so far. A recent study of 485 SQCCs could not provide evidence for a prognostic relevance of keratinisation with respect to overall survival. DFS was not specifically addressed in this study [8]. Our survival results concerning keratinisation in SQCC are somewhat contradictory since in our cohort keratinising SQCC had a worse overall survival compared with nonkeratinising SQCC. This might be partly explained by the association of keratinisation with smaller tumour cell nest sizes and enhanced budding frequency noted in our cohort. However, this observation clearly needs further validation in other large cohorts. Furthermore, we only observed an impact of keratinisation on overall survival but not on DFS, which also needs validation.

Basaloid SQCCs have generally been considered as tumours with a compromised outcome [14-16] and were most recently also characterised as tumours with a specific molecular background [17]. The fraction of

\begin{tabular}{lcc}
$\begin{array}{l}\text { TABLE } 2 \text { Multivariate survival analysis under inclusion of clinicopathological parameters and } \\
\text { tumour grade }\end{array}$ & pazard ratio $(95 \%$-vl) \\
& $1.029(1.010-1.049)$ & 0.003 \\
\hline $\begin{array}{l}\text { Age per year } \\
\text { Sex }\end{array}$ & 1 & 0.097 \\
$\quad$ Female & $1.461(0.934-2.285)$ & $<0.001$ \\
Male & 1 & \\
UICC stage & $1.603(1.101-2.334)$ & \\
1 & $2.738(1.855-4.040)$ & $<0.001$ \\
2 & $4.265(1.312-13.862)$ & \\
3 & $1.561(1.120-2.175)$ & \\
4 & $3.739(2.346-5.959)$ & \\
Grade & & \\
1 & & \\
2 & &
\end{tabular}

UICC: Union for International Cancer Control. 
basaloid SQCC in our cohort is rather small, but the better overall survival outcome of patients with this tumour type in our dataset is noteworthy. Likewise, КАDОTA et al. [8] also found that patients with basaloid SQCCs had a slightly better 5-year overall survival than those with nonbasaloid SQCCs (69\% versus $58 \%$ ). We can only speculate about the reasons for these discrepant data. One explanation for an improved overall survival outcome of resected basaloid SQCCs might be a better response of these tumours to modern adjuvant chemotherapy, possibly due to their high proliferative activity. We tested for this hypothesis in our dataset and found hints that patients with resected basaloid SQCCs treated with adjuvant chemotherapy might fare exceptionally well. However, patient numbers in our subcohorts were clearly far too small to draw any definite conclusions and this topic has not been addressed in any other study. Therefore, this hypothesis certainly has to be validated in follow-up studies specifically focussing on this issue.

We could validate the previously described [8] correlation of cell nest size with overall survival in SQCC. As we observed that nest size is associated with an altered tumour/stroma ratio, we also tested for this criterion by analysing the overall stromal content of our tumours. Indeed, separation of tumours into low, moderate and high stromal content provided a significant prognostic stratification, suggesting that assessment of the tumour/stroma ratio holds further potential as an additional morphological criterion for prognostic SQCC stratification. However, KADOTA et al. [8] could not demonstrate a prognostic effect of the degree of fibrosis in SQCC.

Using the approximate diameter of four average lymphocytes as a cut-off for the categorisation of tumours according to nuclear diameter, the 5-year overall survival of patients with large nuclei (diameter more than four lymphocytes) was reported to be significantly worse than overall survival of patients with small nuclei (diameter less than four lymphocytes) [8]. In our cohort, however, nuclear diameter measured according to this proposal was not associated with patient prognosis.

Taking currently available data on the prognostic value of SQCC morphology into account, we decided to calculate a simple and practical grading scheme based on the two parameters which could be independently validated and which correlated best with patient prognosis, i.e. tumour cell budding and nest size. Factors without successful cross-validation were not included. We disregard proliferative activity as a potential predictor right from the start, as a previous large study from our group firmly established that the association of prognosis and proliferation in SQCC is too complex to be included into a practical grading scheme [9]. By combining the two cross-validated parameters, we established a simple grading algorithm which was highly prognostic. This grading scheme is meant as a first proposal. Clearly, its clinical impact has to be validated in other international large cohorts. In addition, studies on interobserver variations for the contributing histomorphological factors have to be conducted to ensure that such a grading approach is reproducible under real-world diagnostic conditions.

Limitations of our study clearly refer to its retrospective nature and the fact that despite its size, our cohort is somewhat heterogeneous with respect to stage distribution and treatment. However, this also indicates that the proposed grading scheme not only works for selected stages or specific clinical settings.

In conclusion, we addressed the prognostic relevance of specific histomorphological criteria in pulmonary SQCC, and confirmed the previously suggested stage-independent prognostic relevance of tumour cell budding and cell nest size in this entity. On the basis of these data, we suggest a first evidence-based proposal of a clinically relevant grading scheme for SQCC. Our data will hopefully contribute to a success story of truly meaningful histologic grading in pulmonary SQCC, which is able to parallel the tremendous improvements in histology-based classification of pulmonary ADC over the last decade.

\section{References}

1 Travis WD, Brambilla E, Noguchi M, et al. International Association for the Study of Lung Cancer/American Thoracic Society/European Respiratory Society international multidisciplinary classification of lung adenocarcinoma. J Thorac Oncol 2011; 6: 244-285.

2 Kadota K, Nitadori JI, Sima CS, et al. Tumor spread through air spaces is an important pattern of invasion and impacts the frequency and location of recurrences following limited resection for small stage I lung adenocarcinomas. J Thorac Oncol 2015; 10: 806-814.

3 Kadota K, Yeh YC, Sima CS, et al. The cribriform pattern identifies a subset of acinar predominant tumors with poor prognosis in patients with stage I lung adenocarcinoma: a conceptual proposal to classify cribriform predominant tumors as a distinct histologic subtype. Mod Pathol 2014; 27: 690-700.

4 Warth A, Muley T, Kossakowski C, et al. Prognostic impact and clinicopathological correlations of the cribriform pattern in pulmonary adenocarcinoma. J Thorac Oncol 2015; 10: 638-644.

5 Warth A, Muley T, Kossakowski CA, et al. Prognostic impact of intra-alveolar tumor spread in pulmonary adenocarcinoma. Am J Surg Pathol 2015; 39: 793-801.

6 World Health Organization. Classification of tumours. Tumours of the lung, pleura, thymus and heart. Lyon, IARC Press, 2015.

7 World Health Organization. Classification of tumours. Pathology and genetics. Tumours of the lung, pleura, thymus and heart. Lyon, IARC Press, 2004. 
Kadota K, Nitadori J, Woo KM, et al. Comprehensive pathological analyses in lung squamous cell carcinoma: single cell invasion, nuclear diameter, and tumor budding are independent prognostic factors for worse outcomes. J Thorac Oncol 2014; 9: 1126-1139.

9 Warth A, Cortis J, Soltermann A, et al. Tumour cell proliferation (Ki-67) in non-small cell lung cancer: a critical reappraisal of its prognostic role. Br J Cancer 2014; 111: 1222-1229.

10 Karamitopoulou E, Zlobec I, Kolzer V, et al. Proposal for a 10-high-power-fields scoring method for the assessment of tumor budding in colorectal cancer. Mod Pathol 2013; 26: 295-301.

11 Kadota K, Yeh YC, Villena-Vargas J, et al. Tumor budding correlates with protumor immune microenvironment and is an independent prognostic factor for recurrence of stage I lung adenocarcinoma. Chest 2015; 148: 711-721.

12 Maeshima AM, Maeshima A, Asamura $\mathrm{H}$, et al. Histologic prognostic factors for small-sized squamous cell carcinomas of the peripheral lung. Lung Cancer 2006; 52: 53-58.

13 Brandwein-Gensler M, Smith RV, Wang B, et al. Validation of the histologic risk model in a new cohort of patients with head and neck squamous cell carcinoma. Am J Surg Pathol 2010; 34: 676-688.

14 Moro-Sibilot D, Lantuejoul S, Diab S, et al. Lung carcinomas with a basaloid pattern: a study of 90 cases focusing on their poor prognosis. Eur Respir J 2008; 31: 854-859.

15 Moro D, Brichon PY, Brambilla E, et al. Basaloid bronchial carcinoma. A histologic group with a poor prognosis. Cancer 1994; 73: 2734-2739.

16 Brambilla E, Moro D, Veale D, et al. Basal cell (basaloid) carcinoma of the lung: a new morphologic and phenotypic entity with separate prognostic significance. Hum Pathol 1992; 23: 993-1003.

17 Brambilla C, Laffaire J, Lantuejoul S, et al. Lung squamous cell carcinomas with basaloid histology represent a specific molecular entity. Clin Cancer Res 2014; 20: 5777-5786. 